\title{
Gynodioecy in Plantago lanceolata L. V. Frequencies and spatial distribution of nuclear and cytoplasmic genes*
}

Jos M. M. van Damme
Institute for Ecological Research, Department of Dune Research, Weevers' Duin, Duinzoom 20a, 3233 EG Oostvoorne, The Netherlands.

In an old dune grassland the spatial distribution of male sterility type MS1 of Plantago lanceolata was found to be very patchy with spot sizes of generally less than ten square metres. Plants were collected, one per 400 square metres, over a seven hectare area and crossed as males to MS1 plants of known genotypes. Progeny analysis of these crosses and the raising of seed samples, collected from open-pollinated male steriles and hermaphrodites, allowed estimation of the frequencies of nuclear and cytoplasmic male sterility genes. Averaged over the area examined, the recessive and dominant MS1 nuclear genes occurred in frequencies of 0.98 and 0.92 respectively, while the frequency of sterile cytoplasm $R$ was only $0 \cdot 06$. Locally, in and near the MS1 spots, cytoplasm $R$ frequency was higher than average, up to $0 \cdot 39$, and the frequency of the nuclear sterility alleles significantly lower. The MS1 spots probably originated because of both the migration of cytoplasm $R$ individuals into the population and a very low seed dispersal rate. The average as well as the local frequencies of nuclear and cytoplasmic genes can be explained by fitness differences between genotypes, that are associated with the evolutionary conflict between nuclear and cytoplasmic genes.

\section{INTRODUCTION}

There is in the study of the evolution and the maintenance of gynodioecy in higher plants, a direct interaction between theory and experiment. The quality of this interaction depends on the extent to which predictions, arising from the models, are tested experimentally. It has long been assumed that the inheritance of male sterility is determined by either nuclear or cytoplasmic genes. Accordingly, numerous theoretical models have been presented which, because of the simple predictions they made, could fairly easily be tested. When nuclear or cytoplasmic inheritance is assumed to apply, a direct relationship exists between the fitness difference of male steriles and hermaphrodites, required for their coexistence, and the male sterile frequency in the populations (for a review see Van Damme, 1983a). However, the assumption is probably invalid for many, if not all, species. It is suspected that nuclear-cytoplasmic inheritance of male sterility, involving both nuclear and cytoplasmic genes simultaneously, applies to many gynodioecious species

\footnotetext{
* Grassland Species Research Group Publication No. 108. 11081
}

(Ross, 1978; Charlesworth, 1981). Recently it has been shown beyond doubt to exist in Origanum vulgare (Kheyr-Pour, 1980) and in Plantago lanceolata (Van Damme and Van Delden, 1982; Van Damme, 1983b). It is much more difficult to test experimentally models that have been developed from theoretical studies of nuclear-cytoplasmically inherited male sterility (Charlesworth, 1981; Delannay et al., 1981; Ross and Gregorius, 1985; Gouyon, unpubl.). On the one hand this is because maintenance no longer depends on fitness differences between sex phenotypes, but on differences between genotypes; thus a thorough knowledge of the genetics of the system is required prior to making fitness estimates. On the other hand the knowledge of male sterile frequencies is no longer sufficient to test the models, as it is necessary to know the contribution of both nuclear and cytoplasmic genes separately.

Polymorphism for nuclear and cytoplasmic genes has been shown to exist in the field qualitatively (Van Damme and Van Delden, 1982; Ganders, 1978), but actual gene frequencies have not been determined for any species. Determining gene frequencies presents two problems: firstly, the number of nuclear genes for male sterility can be 
relatively large (Van Damme, 1983b), and, secondly, assessing the cytoplasmic type of a plant is usually a laborious task. Even in P. lanceolata, where a (conditional) morphological marker for cytoplasmic type is available, this may take two generations. Relatively simple tests to ascertain the cytoplasmic type, especially those based on restriction endonuclease analysis, exist for several agricultural species (see e.g., Kemble, 1980), but not for wild gynodioecious species.

Another difficulty when comparing model predictions and field data is what should be regarded in the field as the equivalent of a "theoretical" population. As will be shown in this paper, the spatial distribution of male steriles can be very patchy so that their frequency depends strongly on the size of the area examined. Furthermore, the spatial patterns of nuclear and cytoplasmic genes may differ, since the latter are solely dispersed by seeds. Therefore, to determine gene frequencies population structure should be taken into account.

The present paper is one in a series on the maintenance of gynodioecy in $P$. lanceolata. Three male sterility types occur in this species, two of which have been found to have nuclear-cytoplasmic inheritance. One of these, MS1, has been studied here. A plant has the sex phenotype MS1 if it has cytoplasmic type $R$ (two cytoplasmic types have been found, $R$ and $P$ ) and if it is homozygous for two recessive and at least heterozygous for three dominant male sterility genes (Van Damme, $1983 b$ ). Alternatively the five nuclear genes for MS1 can be considered as a set of independent restorer genes, two of which are dominant and three recessive. The partial male sterile, IN 1 , is also expressed in plants with cytoplasm $R$, and results at least partially from incomplete dominance for the recessive MS1 nuclear genes. A very clustered distribution of MS1 and IN1 plants was observed in an old dune grassland, which made it very suitable for examining gene frequencies. Plants from the same population had been used previously in a genetic study (Van Damme, 1983b) and in a study of fitness differences between sex types (Van Damme and Van Delden, 1984).

Firstly, the spatial distribution of sex types in the population was mapped. Secondly, the nuclear gene frequencies in the pollen cloud, which can be imagined hanging above the population, were estimated. Finally, open-pollinated progenies from various years and locations were raised and sexed. The results made it possible to estimate both nuclear and cytoplasmic gene frequencies and to detect spatial differentiation.

\section{MATERIALS AND METHODS}

The field studied is part of an old dune grassland "Westduinen" (Goeree, The Netherlands). It has probably been grazed for over three centuries. Like the rest of the vegetation (height $6 \mathrm{~cm}$ ) the $P$. lanceolata plants are very small. Flowering plant density in the part studied varies from zero to one hundred per square metre. The population biology of $P$. lanceolata has been studied in detail by Van Groenendael (1985). Six different sex types were distinguished: three male sterility types (MS1, MS2, MS3), two partial male sterility types (IN1, IN2, corresponding to MS1 and MS2 respectively) and hermaphrodites $(\mathrm{H})$. Hermaphrodites carry either cytoplasm $R$ or $P$ and are referred to as $\mathrm{H} 1$ and $\mathrm{H} 2$ respectively. For a full account of the genetics of these sex types see Van Damme $(1983 b)$. In July 1981 the numbers of sex types were counted in a $225 \times 350$ square metre area by walking slowly up and down, inspecting a strip of approximately three metres at a time. Each time a group of MS1 or IN1 plant was encountered, its limits were carefully examined. When two MS1 (IN1) plants were less than one metre apart, they were regarded as being from the same spot. Spot size was defined as the number of MS1 (IN1) plants present, and spot area as the smallest circular area containing that group of plants. In various years open pollinated seed samples were taken from the four largest MS1 spots as well as from areas containing no MS1 or IN1 plants. The progeny were raised and scored for sex type as described in Van Damme (1983b). In 1983 the field was divided into $10 \times 17$ squares of $20 \times 20$ square metres. If possible the adult plantain closest to the centre of each square was dug out and transferred to the greenhouse. In ten squares no plantains were found. All but one of the 160 plants flowered in the greenhouse and were scored for sex type. The pollen producing plants among these were to be used as male parents in test crosses with MS1 plants. To this end 12 MS1 plants were each replicated ten times by splitting them into individual shoots after a period of abundant vegetative growth in the garden. The MS1 plants came from two different crosses, both between $\mathrm{H} 1$ and $\mathrm{H} 2$ plants with cytoplasmic type $R$ and $P$ respectively, and yielding only MS1 plants. Five MS1 plants were used from the one cross and had genotype $m r_{1} m r_{1} m r_{2} m r_{2} M r_{3}+$ and were heterozygous at either two $\left(\mathrm{P}=\frac{1}{4}\right)$, one $\left(\mathrm{P}=\frac{1}{2}\right)$ or zero $\left(\mathrm{P}=\frac{1}{4}\right)$ of the $\mathrm{Mr}_{4}$ and $\mathrm{M}_{5}$ loci, $\mathrm{mr}$ and $\mathrm{Mr}$ referring to recessive and dominant MS1 genes respectively (see Van Damme, 1983b). On average, they were heterozy- 
gous at two of the $\mathrm{Mr}$ loci and homozygous dominant at the third. The seven MS1 plants from the other cross were homozygous recessive at the $m r$ loci and at least heterozygous at one of the $\mathrm{Mr}$ loci. It was a priori unknown whether they were heterozygous or homozygous dominant at the two other $M r$ loci. Whenever one of the $120 \mathrm{MS} 1$ shoots flowered in the greenhouse, it was crosssed with one of the field collected hermaphrodites that happened to be flowering. One hundred and sixteen crosses were made, the progenies raised and the sex of the plants scored as described in Van Damme (1983b), except that isolation of the MS1 plants prior to crossing was achieved by keeping them in a separate greenhouse. During the summer of 1984 an average of 23.5 plants (range 17-24) were raised per progeny, of which 22.6 flowered. When expected ratios were tested with chi-square, the correction for continuity was applied when there was one degree of freedom.

\section{RESULTS}

\section{The spatial distribution of MS1}

Averaged over the whole field, (partial) male steriles constitute only a small minority of the population (table 1). The frequencies of the sex types in the field count and in the sample raised in the greenhouse corresponded well. In particular, the frequency of MS1 and IN1 plants, which had received specific attention during the field count, appeared not to be overestimated in the field $\left(\chi_{(1)}^{2}=2 \cdot 32\right)$. The sex types MS2, IN2 and MS3 seemed to be distributed randomly over the field. The distributions of MS1 and IN1, however, coincided completely and were highly clustered. The location of the MS1(IN1) spots is shown in fig. 1. Thirteen groups of type 1 male sterile plants could be distinguished and 24 single plants. Sex type frequencies in the four largest spots are shown separately in table 1 . The figures shown are

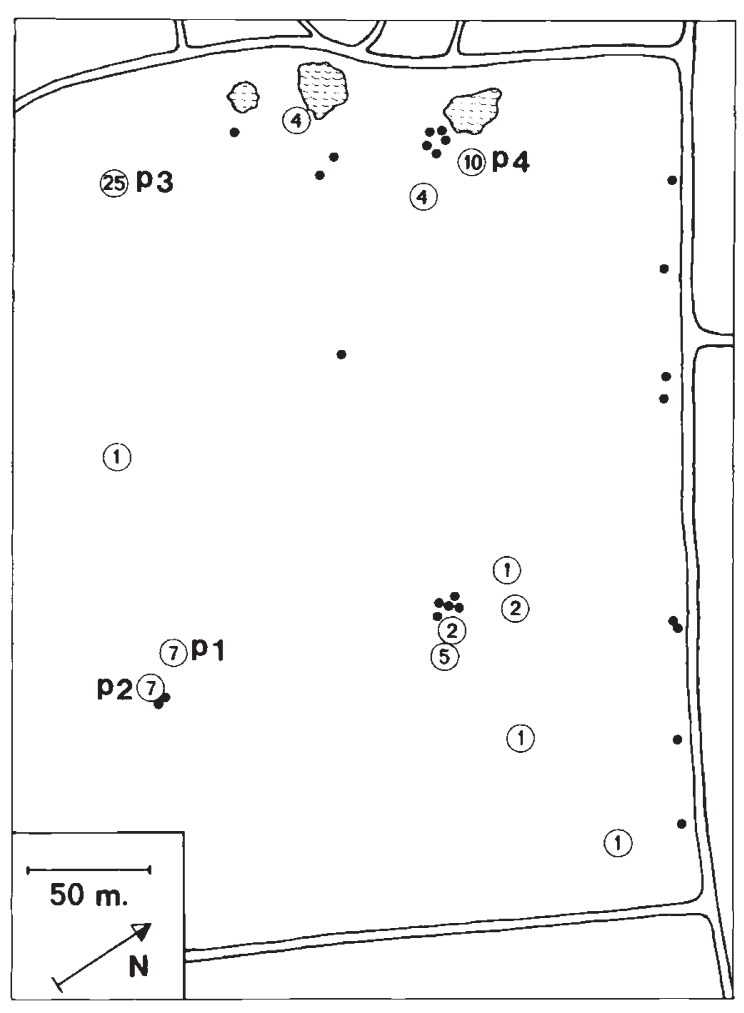

Figure 1 Distribution of spots containing MS1 and IN1 plants of Plantago lanceolata in the Westduinen. Single plants are represented by dots and groups of plants by circles. Within each circle the spot area is given in square metres. The four largest spots are designated $p 1$ to $p 4$. The three shaded areas are dune pools used as watering places by cattle. The spots $p 1$ and $p 2$ correspond with plots 1 and 2 in Van Damme and Van Delden (1984).

averages: the frequency of MS1 is close to zero at the borders and rises to 60 per cent in the centre of the spots. Even within the spots, hermaphrodites are predominant. The spots are more or less circular, except for $p 3$, which has the appearance of two barely overlapping circles. The nine smaller spots have areas ranging from one to five square

Table 1 Distribution (\%) of sex types of Plantago lanceolata in the Westduinen. For the location of samples p1-p4 see fig. 1

\begin{tabular}{llllllll}
\hline & \multicolumn{2}{l}{ Sex type } & & & \\
\cline { 2 - 6 } Sample description & MS1 & IN1 & MS2 & IN2 & MS3 & H & Sample size \\
\hline Field count, total & 4.6 & 0.6 & 0.5 & 0.9 & 0.5 & 92.9 & 6902 \\
p1 & 28.6 & 5.4 & 0 & 0 & 0 & 66.1 & 112 \\
p2 & 25.6 & 1.6 & 0 & 0 & 0 & 72.8 & 188 \\
p3 & 21.3 & 3.0 & 0.3 & 1.2 & 0 & 74.3 & 334 \\
p4 & 22.7 & 1.6 & 0 & 4.7 & 0 & 71.1 & 128 \\
remainder & 1.9 & 0.4 & 0.6 & 0.9 & 0.5 & 95.7 & 6140 \\
Field sample raised in greenhouse & 2.5 & 0 & 0.6 & 3.1 & 0.6 & 93.2 & 159 \\
\hline
\end{tabular}


metres, with on average five MS1(IN1) individuals per square metre. The single MS1 plants are mostly scattered near the spots and along the NE road. The latter may well be immigrants from a $P$. lanceolata population along the road just outside the fence. This road population consists of a few hundred plants which in contrast to the Westduinen plants, are vigorous and of which 19.3 per cent $(n=135)$ are MS1 or IN1.

The structure of the population, shown in fig. 1 , suggests in itself a particular genetic composition, that would arise in a population consisting entirely of plants with the "non-sterile" cytoplasm $P$ and with sterility alleles at all MS1 nuclear loci. If for instance a MS1 plant were to migrate into this hermaphrodite population, its progeny would consist entirely of MS1 plants as all the pollen, despite its fertile phenotype, has a MS1-sterile genotype. Provided the seed dispersal rate is low, a MS1 spot could be established in this way. A comparatively easy way of testing this idea is by sampling the genetic composition of the pollen produced by the population.

\section{Gene frequencies among hermaphrodites}

In order to examine the genetic composition of the pollen producing plants in the population, plants were collected in the field and used to fertilise MS1 plants. A survey of the results is given in fig. 2(a). More than half of the crosses gave only MS1 plants as progeny and all of the crosses more than 35 per cent MS1. Clearly the frequencies of the sterility alleles at the MS1 loci are high, or, expressed differently, the frequencies of the restorer alleles are low. Particularly the absence of
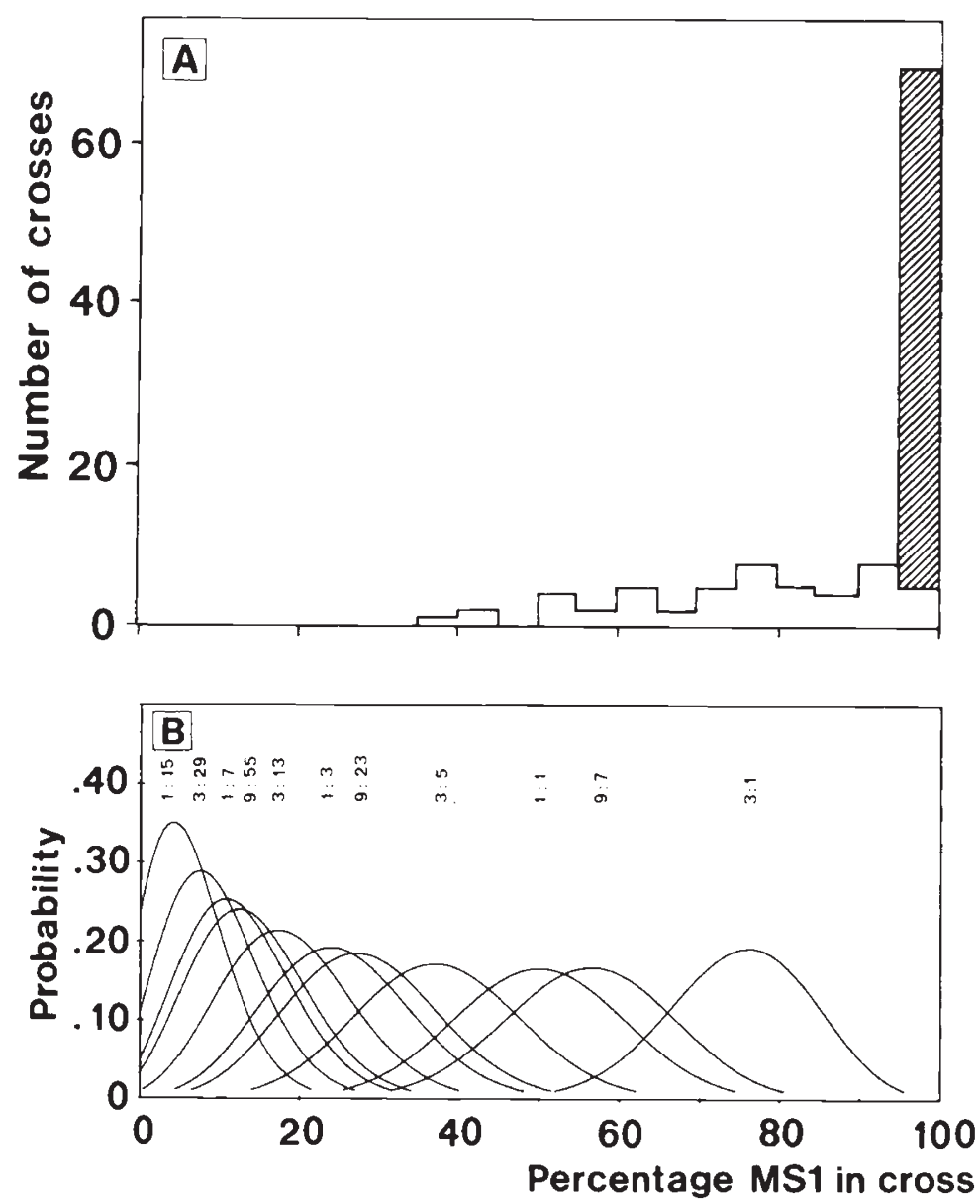

Figure 2 Frequency distribution of the percentage MS1 plants in the progenies of MS1 $\times$ H crosses: (a) The observed distribution. The shaded bar represents crosses yielding only MS1 plants. (b) Theoretical distributions, calculated for all possible MS1: not-MS1 ratios in the model under study (see text), except for $1: 0$ and $0: 1$, and based on the average progeny size of $22 \cdot 6$ plants. Each distribution covers a unit area. 
crosses without MS1 plants in their progeny shows that none of the male parents were homozygous for the restorer allele at either of the $m r$ loci.

In order to make quantitative estimates of the gene frequencies, the crosses were divided according to their MS1: not-MS1 ratio. In the Appendix it is explained that with the genetic model used, 13 different ratios are possible (fig. 2(b)). In view of the considerable overlap of most of the probability curves in fig. 2(b), only the extreme ratios $1: 0$ and $0: 1$, as well as the $3: 1$ ratio can be reasonably well distinguished from each other. The procedure for dividing the crossses into ratio groups included the following criteria:

-the MS1 expression in known to be slightly unstable. The size limit of the $1: 0$ ratio group was assessed by taking the MS1 instability expression into account and by assuming that MS1 genotypes never show a $\mathrm{H}$ phenotype (Van Damme, 1983b).

-crosses, placed in the 3:1 group, should be homogeneous (according to chi-square analysis) with respect to MS1 : not-MS1 segregation. Furthermore, as a group they should fit the $3: 1$ ratio. The crosses in this group should only occasionally show $\mathrm{H}$ plants in their progeny, which, when occurring, are supposed to result from instability of the IN1 genotypes (Van Damme, 1983b).

Firstly, the maximum size of the $3: 1$ group was established. Then the minimum size of this group was obtained by setting the $1: 0$ group at a maximum, and then leaving out crosses of the $3: 1$ group with the lowest MS1 fraction in their progeny, until the resulting group fitted the $3: 1$ ratio again. The resulting size range was $21-35$ crossses. As a fair size the average was taken (table 2). Consequently the sizes of the 1:0 group and the rest group are also known. The 1:0 group contains 76 crosses, of which 14 segregated one or two IN1 plants. The instability percentage was $1 \cdot 0$, while in a previous study it was $2 \cdot 8$ (Van Damme, $1983 b$ ). The rest group of 12 crosses appeared to be homo- geneous with respect to MS1:not-MS1 segregation (table 2). Although it is referred to in table 2 as a $1: 1$ ratio group; it fitted both $1: 1$ and $9: 7\left(\chi_{(1)}^{2}=\right.$ 0.53 and 1.51 respectively) while the crosses, when tested individually, fitted several other ratios shown in fig. 2(b).

The $3: 1$ ratios in table 2 can occur only if both male and female parent in the cross are heterozygous at one and the same $\mathrm{Mr}$ locus. Of the $12 \mathrm{MS} 1$ genotypes used as female parents, five were, on average, heterozygous at two $\mathrm{Mr}$ loci, and seven at least at one locus. Since both groups of MS1 parents appeared not to differ noticeably in the fraction of their progenies with a $3: 1$ ratio $(7 / 33$ and $21 / 83$ respectively, $\chi_{(1)}^{2}=0 \cdot 22$ ), they can be considered as having the same ability to reveal restorer alleles at the $M r$ loci in male parents. As is shown in the Appendix, the results in table 2 permit estimates of the average restorer allele frequencies at the $M r$ and $m r$ loci. They appear to be $0.017 \pm 0.009$ and $0.084 \pm 0.017$ respectively. Provided these estimates hold for each of the loci involved, in particular for the $\mathrm{Mr}$ loci (since these have only partly been sampled), the expected frequencies of sex types among the male parents of the test crosses can be calculated, using the inheritance model of Van Damme (1983b) and assuming all male parents have cytoplasm $R$. It appears that in this case 99.5 per cent of the plants should show partial or complete type 1 male sterility. Since no MS1 or IN1 plants were present among the male parents used, the frequency of cytoplasm $R$ among these plants is apparently very low. Thus, in conclusion, averaged over the whole field the frequencies of the restorer alleles at both the $M r$ and $m r$ loci are close to zero, whereas the frequency of the "non-sterile" cytoplasm $P$ is close to unity.

\section{Spatial distribution of MS1 genes}

The spatial distribution of the male parents, grouped according to the MS1: not-MS1 ratio in their progenies, is shown in fig. 3. There is no

Table 2 Distribution (in numbers) of sex types in the progenies of MS1 $\times \mathrm{H}$ crosses. The progenies have been grouped according to their presumed MS1: not-MS1 ratio. Sex type IN1 is subdivided: a means predominantly male sterile, $c$ predominantly hermaphrodite and $b$ intermediate between $a$ and $c$

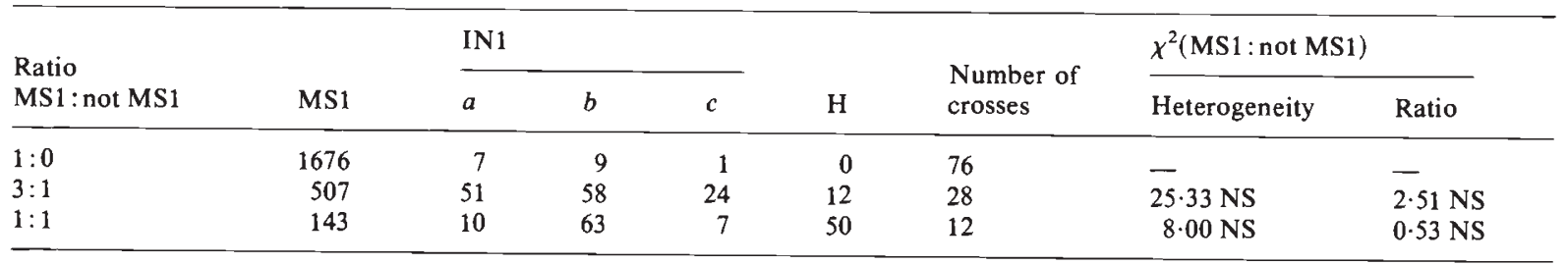



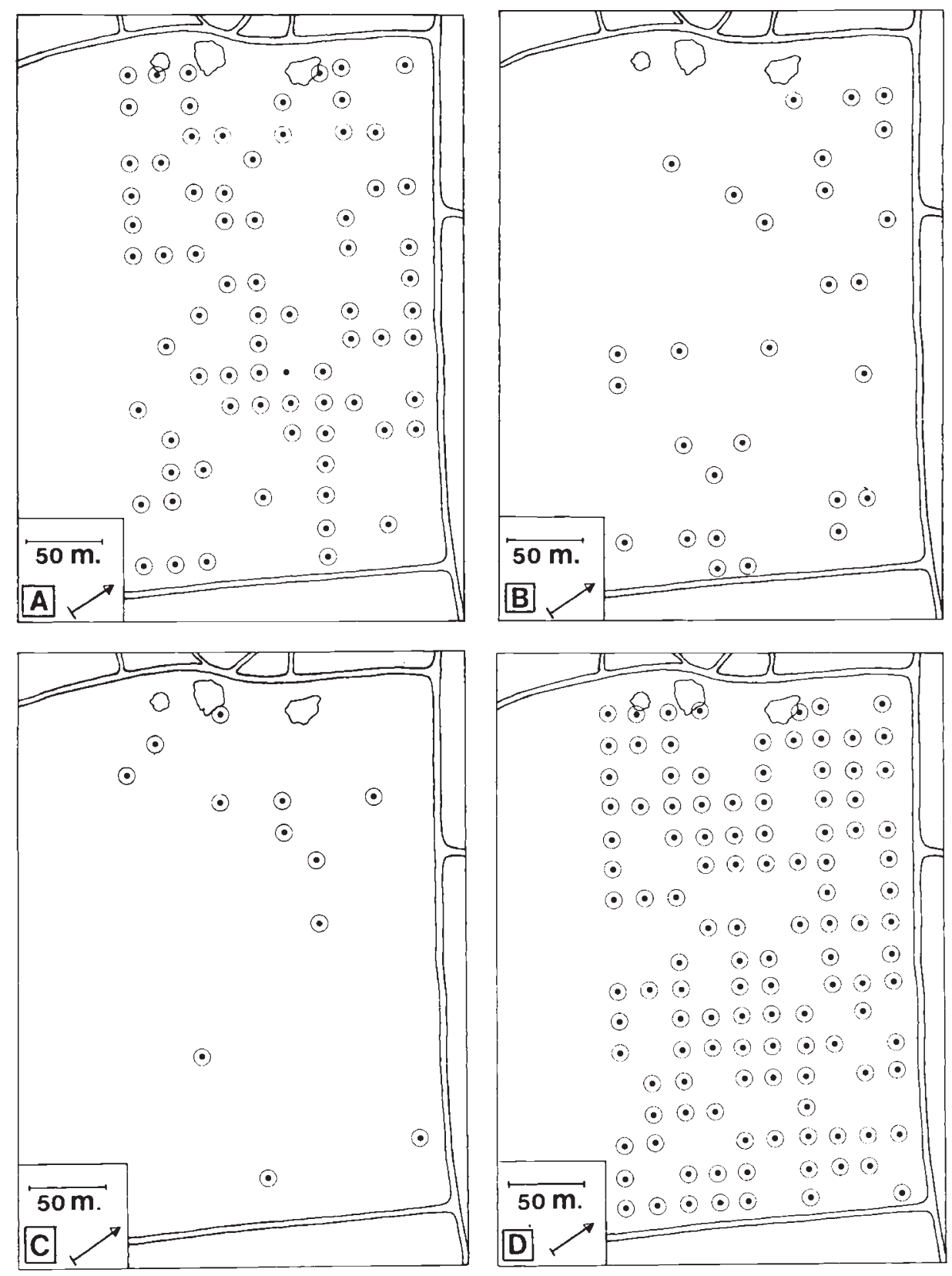

Figure 3 Spatial distribution of male parents of test crosses giving particular MS-: not MS1 ratios: (a) 1:0, (b) 3:1, (c) 1:1, and (d) the distribution of all parents.

significant clustering of male parents with a particular ratio in any part of the field, not even when plants near or in the MS1 spots are compared to the rest (cf. fig. 1). This is not surprising since for the crossing experiment only one plant was collected per 400 square metres, whereas the largest MS1 spot was 25 square metres, most of them being less than ten. In order to know whether the genetic composition of the plants in and near the MS1 spots differed from the rest of the population, the MS1 plants growing in the spots were used. These plants sample the local pollen cloud during flowering, thereby imitating the above crossing experiment. The information obtained by raising the open pollinated progenies of the MS1 plants is, however, less than that from the crossing experi- 
ment. Firstly, because it concerns a mixture of full and half sib instead of purely full sib families, and secondly because the genotypes at the $\mathrm{Mr}$ loci of the female parents in the spots are less well known than in the crossing experiment. Nevertheless, a qualitative comparison can be made between the seed samples collected from the MS1 plants in the four largest spots and the grand total of the crossing experiment (table 3 ). Only the $p 4$ sample has a sex type distribution comparable to the grand total $\left(\chi_{(2)}^{2}=1 \cdot 10\right)$. The samples from $p 1, p 2$ and $p 3$ all differ significantly $(P<0.001)$ from both $p 4$ and the grand total, but are homogeneous among themselves $\left(X_{(6)}^{2}=5 \cdot 33\right)$. The apparently enhanced frequencies of the restorer alleles in these spots could be due to a higher ability of the MS1 plant therein to reveal restorer alleles. However, if all the MS1 parents in the spots $p 1, p 2$ and $p 3$ are assumed to have the most "restorer-allele-sensitive" genotype (i.e., homozygous at the $m r$ and heterozygous at all $\mathrm{Mr}$ loci), the expected percentage MS1 plants in their open pollinated progeny can be calculated to be $85 \cdot 0$. For this calculation the estimated restorer allele frequencies at the $m r$ and $M r$ loci of the preceding section have been used. The MS1 frequencies in the $p 1, p 2$ and $p 3$ samples of MS1 parents in table 3 then appear to be all significantly lower $(P<0.001)$ than expected. It therefore can be concluded that the spots $p 1, p 2$ and $p 3$, in contrast to $p 4$, have higher frequencies of restorer alleles than average.

Seed samples have also been collected from $\mathrm{H}$ plants, both within and between MS1 spots (table
3). The almost total absence of MS1 and IN1 plants in the samples taken from between the spots is in accordance with expectation, since it can be concluded from the preceding sections that the plants present between the MS1 spots are nearly all $\mathrm{H} 2$ plants with cytoplasm $P$. The samples from the central area apparently included a small MS1 spot (cf. fig. 1). The high degree of correspondence between the samples of $\mathrm{H}$ plants within and between the spots shows the frequency of $\mathrm{H} 1$ plants, with cytoplasm $R$, among $\mathrm{H}$ plants within the spots to be low as well. Their presence should be expressed by MS1 or IN1 plants in the samples, since it is known that in this population $\mathrm{H} 1$ plants, when crossed with $\mathrm{H} 2$ plants, segregate $25-100$ per cent MS1 (Van Damme, 1983b). A rough estimate of the frequency of $\mathrm{H} 1$ among $\mathrm{H}$ plants in the MS1 spots can be made by assuming that each of the 53 parents of the 1979 p2 sample in table 3 contributed equally to the seed sample. Taking the above range in segregation ratios into account, it can then be calculated that $2 \cdot 2-8 \cdot 6$ parents of the p2 sample should have been $\mathrm{H} 1$. This is $2-8$ per cent of all $\mathrm{H}$ plants tested from the MS1 spots (table 3), indicating that the field frequency of $\mathrm{H} 1$ plants should be approximately the same as that of IN1 plants (table 1). Equal IN1 and $\mathrm{H} 1$ frequencies are also found in the open pollinated progenies of MS1 plants (table 3), which, in view of their frequencies in the spots (table 1) are the main suppliers of these phenotypes.

Finally, local frequencies of cytoplasmic types can be estimated. Setting the frequency of cyto-

Table 3 Distribution (\%) of sex types of Plantago lanceolata in seed samples, collected at various locations in the Westduinen (see Fig. 1). The year of sampling and the number of seed parents contributing to each sample are also given

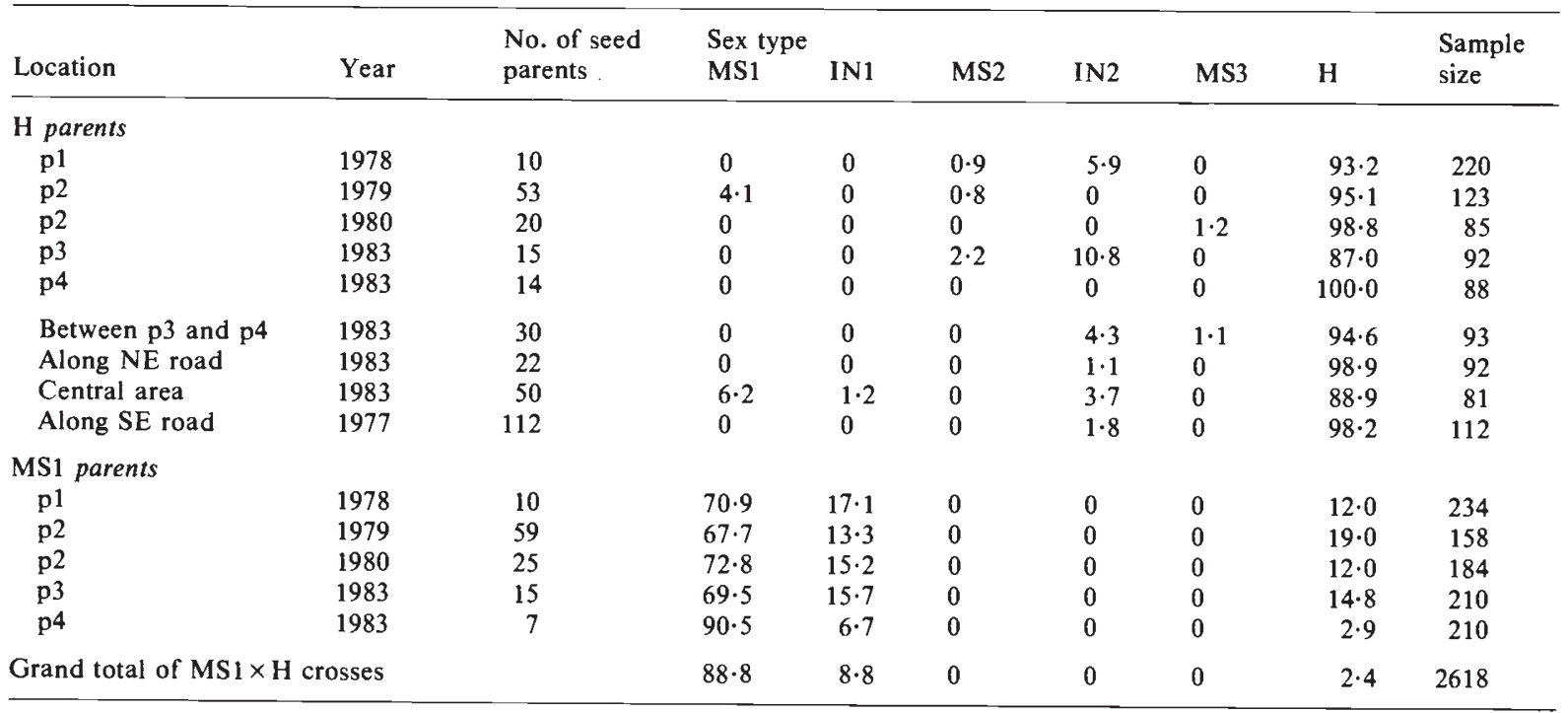


plasm $R$ equal to the frequency of MS1 plus two times the IN1 frequency, its value varies among the four largest spots from 26-39 per cent. In conclusion, there is considerable spatial differentiation with respect to both nuclear and cytoplasmic genes determining MS1 in the Westduinen field.

\section{DISCUSSION}

The crossing results support earlier findings with respect to the genetics of MS1 (Van Damme, $1983 \mathrm{~b}$ ). The occurrence of $3: 1$ ratios exclude the possibility that only recessive MS1 genes are involved. One dominant MS1 gene can account for all ratios, but does not give a satisfactory fit, nor do two genes $(P<0.005$ in both cases $)$. The minimum required is one recessive and one dominant MS1 gene, which confirms the existence of $\mathrm{mr}$ and $\mathrm{Mr}$ loci. In contrast to Van Dijk's (1985a) findings, the present results confirm the genetic determination of IN1 by complete restoration at one of the $\mathrm{Mr}$ loci with all other loci in the sterile condition (see the 3:1 results in table 2). Indirect support for the genetic determination of MS1 and IN1 by the same genes comes from their completely coinciding distributions in the field (fig. 1).

The distribution of nuclear and cytoplasmic genes determining the MS1 sex type in the Westduinen field, is clearly not at random. The largest part of the area is fixed by the "non-sterile" cytoplasm $P$ and has frequencies of the restorer alleles at the $m r$ and $M r$ loci which are close to zero. The "sterile" cytoplasm $R$ does occur in 5-6 per cent of the plants, but these are concentrated in small spots scattered over the area and covering only $0 \cdot 1$ per cent of the surface. Furthermore, in three of the largest spots the restorer allele frequencies of the nuclear genes appear to be higher than average. It seems likely that the MS1 spots were founded after migration of an individual with cytoplasm $R$ into the cytoplasm $P$ area. If this individual is MS1, its progeny will be largely MS1 due to the high frequencies of the sterility alleles at the nuclear loci of cytoplasm $P$ plants (see spot $p 4$ and grand total in table 3 ). Since MS1 plants in this population have an advantage over $\mathrm{H}$ plants in seed production, seed weight and survival rate (Van Damme and Van Delden, 1984), they, and therefore cytoplasm $R$, will spread. Due to the low seed dispersal rate in the Westduinen $(8 \mathrm{~cm} /$ year, Bos et al. 1985), patches of plants with cytoplasm $R$ are formed, which are visible because the great majority of these plants are MS1. If the first cyto- plasm $R$ plant is not MS1, the process is likely to be the same, but to develop more slowly. The results in table 3 suggest a further development via spot $p 4$ to the three other large spots, implying an increase of the restorer allele frequencies after the initial increase of cytoplasm $R$.

This sequence of events follows the first part of the scheme proposed by Gouyon and Couvet (1985), who consider the development of a gynodioecious population to result from an evolutionary conflict between nuclear and cytoplasmic genes. According to this scheme, the evolution of a gynodioecious population starts with an invasion of MS individuals with only sterility alleles at the nuclear loci, into a population of plants that all have these alleles as well, and in addition "nonsterile" cytoplasm. In a later stage restorer alleles invade the population, probably by migration. Due to the fitness relations their frequency rises locally in the population wherever the "sterile" cytoplasm frequency is sufficiently high. This scheme seems to apply to the Westduinen situation if an area of about a few square metres is taken as the "population", which is close to the neighbourhood area in this field (Bos et al., 1985; Van Dijk, 1985b). However, there is a difference. All over the Westduinen, restorer alleles are present at a low frequency. It therefore seems likely that they do not invade the MS1 spots, but that, on the contrary, a spot expands until it runs into restorer alleles. Alternatively, restorer alleles may be present from the start of spot formation, when the founder plant carries alleles with it, even when it is MS1, since at three of the five MS1 loci the restorer alleles are recessive.

An argument in favour of this refinement can be found when considering fitnesses. The low frequencies of restorer alleles at both the $m r$ and $\mathrm{Mr}$ loci among cytoplasm $P$ plants suggest these alleles have a selective disadvantage at the genotypic level compared to the sterility alleles in the cytoplasm $P$ area. This fitness difference is apparently small enough to allow restorer alleles to remain present for a long time at low frequencies, but will prevent their spreading in the cytoplasm $P$ area. The enhanced frequencies of restorer alleles in some of the MS1 spots (table 3) suggests that the fitness relations are different here, which is at least partly caused by male sterility of particular genotypes in cytoplasm $R$ plants. If the assumption by several authors (Ross and Gregorius, 1985; Gouyon and Couvet, 1985; Gouyon, unpublished) holds, that the restorer alleles become fixed when only the "sterile" cytoplasm, here $R$, is present, then their frequency will 
rise in the MS1 spots, as observed, but only after the $R$ frequency has become sufficiently high. Thus there would be a time lag between the appearance of the MS1 spots and the increase of restorer allele frequencies. This may explain the observed difference between spot $p 4$ and the other large spots in table 3 . A time lag would also occur if the founder plant were to bring some restorer alleles with it.

The further development of the MS1 spots is unknown. Even the above interpretation should be considered with some caution, since the spots in fig. 1 were assumed to represent a particular development according to their size and genetic composition, whereas the period of observation was relatively short. The half life of plantains at the Westduinen is about two years for hermaphrodites (Van Damme and Van Delden, 1984; Van Groenendael, 1985) and seed dispersal is very low (Bos et al., 1985), so that changes in spatial patterns require considerable time. Spot $p 4$ for example, might in due course appear to be part of an older, larger spot that is falling apart, instead of a relatively young spot (fig. 1). Of particular interest is the question whether the spots will ever reach a state of equilibrium. An indication that the three spots $p 1, p 2$ and $p 3$ might be in equilibrium is that their genetic compositions are very alike. This is expressed in the homogeneity of the open pollinated progenies of MS1 plants in spite of variation in year and site of sampling (table 3).

The present study makes it likely that heterogeneity in fitness exists among $\mathrm{H}$ genotypes as was predicted from theoretical studies. In particular, computer studies have made it plausible that a higher fitness of the sterility alleles relative to the restorer alleles in the presence of cytoplasm $P$ is a necessary condition for joint polymorphism (Charlesworth, 1981; Delannay et al., 1981). In analytical studies this difference was taken as an assumption (Delannay et al., 1981; Gregorius and Ross, 1984; Ross and Gregorius, 1985) and it is required if the evolution of a gynodioecious population is to proceed as a result of a conflct between nuclear and cytoplasmic genes, as proposed by Gouyon and Couvet (1985). None of the theoretical studies predict precise frequencies of trajectories towards equilibrium. The number of possible solutions in nuclear-cytoplasmic models is immensely large, even if they relate to a single nuclear gene and two cytoplasmic types, and analytical solutions are not available at present. The situation in P. lanceolata at the Westduinen provides one example of how microevolution of a gynodioecious population might occur.
Acknowledgements Part of this study was conducted at the Institute of Genetics of the University of Groningen, and financed by a BION-ZWO grant. I wish to thank P. H. Gouyon for showing me his unpublished results and P. H. Gouyon, F. Kjellberg, G. Valdeyron, P. van Tienderen and J. Woldendorp for critically reading an earlier draft of the manuscript.

\section{REFERENCES}

BAILEY, N. T.1951. Testing the solubility of maximum likelihood equations in the routine application of scoring methods. Biometrics, 7, 268-274.

BOS, M., HARMENS, H. AND VRIELING, K. 1985. Gene flow in Plantago I Dispersal and neighbourhood in P. lanceolata. Heredity, in press.

CHARLESWORTH, D. 1981. A further study of the problem of the maintenance of females in gynodioecious species. $\mathrm{Her}$ edity, 46, 27-39.

DELANNAY, X., GOUYON, P. H., AND VALDEYRON, G. 1981 Mathematical study of the evolution of gynodioecy with cytoplasmic inheritance under the effect of a nuclear restorer gene. Genetics, 99, 169-181.

GANDERS, F. R. 1978. The genetics and evolution of gynodioecy in Nemophila menziesii (Hydrophyllaceae). Can. J. Bot., $56,1400-1408$.

GOUYON, P. H. AND COUVET, D. 1985. Selfish cytoplasm and adaptation: variations in the reproductive system of Thyme. In: Haeck, J. and Woldendorp, J. W. (eds.). Phenotypic and geotypic variation within and between plant populations. Verh. Kon. Ned. Akad. Wet. afd. Natuurkd 2e Reeks, 85. North Holland Publ. Comp. Amsterdam.

GREGORIUS, H. R. AND ROSS, M. D. 1984. Selection with genecytoplasm interactions. I Maintenance of cytoplasm polymorphisms. Genetics, 107, 165-178.

KEMBLE, R. J. 1980. A rapid, single leaf assay for detecting the presence of " $S$ "'-male-sterile cytoplasm in maize. Theor. Appl. Genet., 57, 97-100.

KHEYR-POUR, A. 1980. Nucleo-cytoplasmic polymorphism for male sterility in Origanum vulgare L. J. Hered., 71, 253-260.

Ross, M. D. 1978. The evolution of gynodioecy and subdioecy. Evolution, 32, 174-188.

ROSS, M. D. AND GREGORIUS, H. R. 1985. Selection with genecytoplasm interactions. II Maintenance of gynodioecy. Genetics, 109, 427-439.

VAN DAMME, J. M. M. $1983 a$. On gynodioecy in Plantago lanceolata. Thesis Univ. of Groningen.

VAN DAMME, J. M. M. 1983 b. Gynodioecy in Plantago lanceolata L. II Inheritance of three male sterility types. Heredity, $50,253-273$

VAN DAMME, J. M. M. AND VAN DELDEN, W. 1982. Gynodioecy in Plantago lanceolata L. I Polymorphism for plasmon type. Heredity, 49, 303-318.

VAN DAM ME, J. M. M. AND VAN DELDEN, W. 1984. Gynodioecy in Plantago lanceolata L. IV Fitness components of sex types in different life cycle stages. Evolution, 38, 1326-1336.

VAN DIJK, H. 1985a. Allozyme genetics, self-incompatibilty and male sterility in Plantago lanceolata. Heredity, 54, 53-64.

VAN DIJK, H. 1985b. Geneti. variability in Plantago species in relation to their ecology. Thesis Univ. of Groningen.

VAN GROENENDAEL, J. M. 1985. Selection for different life histories in Plantago lanceolata. Thesis Univ. of Nijmegen. 


\section{APPENDIX}

The MS1 plants used as female parents in the test crosses were homozygous recessive at the two $\mathrm{mr}$ loci and, on average, heterozygous at two $\mathrm{Mr}$ loci. A $\mathrm{Mr}$ locus that is homozygous dominant in the female parent does not give any information in a test cross, since the progeny is always MS1 as far as the contribution of this locus is concerned. Therefore restorer alleles in the male parents can be detected at both $m r$ loci and at two $\mathrm{Mr}$ "equivalents". The latter represents all $\mathrm{Mr}$ loci, although the relative contribution of each is not known. The probability however, that one of the $\mathrm{Mr}$ loci does not contribute at all equals the probability that all 12 MS1 parents are homozygous at the same $M r$ locus and is very low $\left(P<10^{-4}\right)$.

In the following it is assumed that all genotypic frequencies are at Hardy-Weinberg equilibrium. Further, since there are only two degrees of freedom (see below) the restorer alleles of the $\mathrm{Mr}$ loci will be considered to have the same frequency $(p)$; the same holds for the restorer allele frequencies (q) of the $m r$ loci.

Per $m r$ locus the possible MS1 : not-MS1 ratios are $1: 0,1: 1$ and $0: 1$, and per $M r$ locus $1: 0,3: 1$ and $1: 1$. When the four loci are put together, 13 ratios are possible (Fig. 2(b)) of which only two can be used. The expectations are:

$\begin{array}{ccc}\text { Ratio } & \text { Expectation } & \text { Observed } \\ 1: 0 & n(1-p)^{4}(1-q)^{4} & a \\ 3: 1 & 4 n p(1-p)^{3}(1-q)^{4} & b \\ \text { all others } & n\left(1-(1-p)^{3}(1+3 p)(1-q)^{4}\right) & c\end{array}$

where $a, b$ and $c$ are the numbers of crosses showing a particular MS1: not MS1 ratio and $n=$ $a+b+c$. A maximum likelihood solution is obtained by putting expectations equal to the observed value (Bailey 1951). Dividing (1) by (2) gives $\hat{p}=b /(4 a+b)$ and inserting $\hat{p}$ in (3) yields $\hat{q}=1-\frac{1}{4}(4 a+b) n^{-1 / 4} a^{-1 / 4}$. Variances can be obtained by applying Fisher's formula:

$$
\operatorname{var}(T)=\sum_{i=1}^{k}\left(\frac{\partial T}{\partial a_{i}}\right)_{0}^{2} n p_{i}-n\left(\frac{\partial T}{\partial n}\right)_{0}^{2}
$$

where the estimator $T$ is based on $n$ observations, which are distributed among $k$ classes, the $i$ th of which contains $a_{i}$ observations with expectations $n p_{i}$. This results in

$$
\operatorname{var}(\hat{p})=16 a b(a+b) /(4 a+b)^{4} \text {, }
$$

and

$$
\operatorname{var}(\hat{q})=\frac{(3 b-4 a)^{2}+16 a b}{256 a^{2} \sqrt{a n}}-\frac{(4 a+b)^{2}}{256 a n \sqrt{a n}} .
$$

By putting $a=76, b=28$ and $n=116$ the estimates are $\hat{p}=0.084 \pm 0.017$ and $\hat{q}=0.017 \pm 0.009$. A chisquare test on observed and expected values shows that the fit is satisfactory $\left(\chi_{(2)}^{2}<10^{-4}\right)$. 